\title{
The anti-inflammatory effects of macrolides
}

\author{
Debbie Wales, Mark Woodhead \\ Department of Respiratory Medicine, Manchester Royal Infirmary, Manchester, UK
}

Introductory article

\section{Erythromycin and clarithromycin attenuate cytokine-induced endothelin-1 expression in human bronchial epithelial cells}

\begin{abstract}
H Takizawa, M Desaki, T Ohtoshi, S Kawasaki, T Kohyama, M Sato, J Nakajima, M Yanagisawa, K Ito
Erythromycin and its fourteen-member macrolide analogues have attracted attention for their efficacy $\stackrel{\circ}{3}$ in bronchial asthma. However, their mechanisms of action remain unclear. We evaluated the effects of $\vec{\nabla}$ the macrolide antibiotics on endothelin-1 (ET-1) expression in normal and transformed human bronchial $\mathbb{0}$ epithelial cells, one of the sources of this potent bronchoconstrictor important in the pathogenesis of $\frac{}{\square}$ asthma. Human bronchial epithelial cells were obtained from the resected bronchi, and the effect of $\overrightarrow{0}$ several antimicrobial and antiasthmatic drugs on the production and messenger ribonucleic acid ( $m R N A) \Theta$ levels of ET-1 was evaluated. Bronchoepithelial cells were also isolated from the mucosa of asthmatic patients under fibreoptic bronchoscopy, and the modulating effects of the drug were studied. Erythromycin and clarithromycin uniquely suppressed mRNA levels as well as the release of ET-1 at $\overline{0}$ therapeutic and non-cytotoxic concentrations (percentage inhibition of ET-1 protein release: $26.4 \pm 5.22 \%$ and $31.2 \pm 7.45 \%$, respectively, at $10^{-6} \mathrm{M}$ ). Furthermore, erythromycin and clarithromycin inhibited ET1 expression in bronchoepithelial cells from patients with chronic, stable asthma. A glucocorticosteroid, dexamethasone, also inhibited ET-1 expression. In contrast, theophylline, salbutamol and FK506 had $\equiv$ no effect on ET-1 production. Our findings demonstrated that these fourteen-member macrolide antibiotics had an inhibitory effect on endothelin-1 expression in human bronchial epithelial cells. Moreover, this new mode of action may have some relevance to their clinical efficacy in bronchial asthma. (Eur Respir J 1998;12:57-63)
\end{abstract}

Macrolide antibiotics are widely used in the treatment of infection. They show broad spectrum antibacterial activity against Gram positive bacteria-for example, Streptococcus pneumoniae - and intracellular bacteriafor example, Mycoplasma pneumoniae, Chlamydia and Legionella species-and combine this with good tissue penetration. It has been known for many years that macrolide antibiotics have an effect on host cell function as well as an antimicrobial effect. Erythromycin and troleandomycin were shown to improve the clinical status of patients with steroid dependent asthma over 20 years ag $\phi^{1}$ and, more recently, long term low dose erythromycin has been shown to reduce bronchial hyperreactivity.2 However, the mechanisms by which these effects occur have remained obscure. In the Introductory Article by Takizawa et al erythromycin and clarithromycin have been shown to suppress endothelin-1 expression and release by human bronchoepithelial cells which may provide new insight into how this effect is achieved.

Endothelin-1, a polypeptide, is the most potent vasoconstrictor know and also has potent bronchoconstrictor effects. It has been reported to stimulate mucus secretion and to cause mucosal oedema. ${ }^{7}$ In experimental work it has been shown to play a key role as a mediator of airway inflammation. A considerable 음 increase in the concentration of endothelin-1 in $\bar{N}$ bronchoalveolar lavage (BAL) fluid of the rat occurred of during the early phase of experimental inflammation 0 and this was associated with a rise in the total cell, $\mathrm{C}$ eosinophil, and neutrophil counts. Treatment with an endothelin-1 receptor antagonist inhibited the increase $\bullet$ in BAL fluid eosinophils and reduced the inflammatory $\mathbb{\Phi}$ reaction in the lung tissue. Bronchial smooth muscle + cells have been shown to possess specific binding sites $\frac{7}{0}$ for endothelin- ${ }^{9}$ and the bronchial epithelial cells of $\overrightarrow{\mathbb{D}}$ asthmatic patients express preproendothelin-1 mRNA $\stackrel{\odot}{\mathbb{Q}}$ and release large amounts of biologically active endo- $\Omega$ thelin-1 10 Corticosteroids have been shown to reduce the production of endothelin-1 and, for the first time, of this effect has been shown by macrolides.

In this study a clinical role for endothelin-1 as a 흑 bronchoconstrictor was suggested by a negative correlation with peak expiratory flow rate; surprisingly, no relationship was found with forced expiratory volume in one second $\left(\mathrm{FEV}_{1}\right)$ but the number of subjects studied was small. A number of antimicrobial and anti-asthma drugs were also tested but inhibition of endothelin-1 


\begin{tabular}{ll} 
Table 1 Macrolides \\
\hline Ring structure & Name \\
\hline 14-membered & $\begin{array}{c}\text { Erythromycin, clarithromycin, roxithromycin, } \\
\text { dirithromycin }\end{array}$ \\
$\begin{array}{l}\text { 15-membered } \\
16 \text {-membered }\end{array}$ & $\begin{array}{c}\text { Azithromycin } \\
\text { Josamycin, spiramycin }\end{array}$ \\
\hline
\end{tabular}

release was found to occur only with the 14-membered ring macrolides erythromycin and clarithromycin. Interestingly, no such effect was seen with josamycin, a 16-membered ring macrolide, or with FK506 (table 1).

An effect on endothelin- 1 is one of the most recently described of a number of anti-inflammatory properties shown by the 14-membered ring macrolides (fig 1). The following sections describe some of these mechanisms and lead on to a description of other studies of the use of macrolides in asthma and other conditions characterised by inflammation.

\section{Macrolides and the inflammatory response}

The inflammatory process is multifactorial and macrolides have been shown to act in a number of different ways, their effects being demonstrated in various animal models (table 2). Oedema produced by the injection of carrageenin into the paw of a rat can be suppressed by pretreatment of the animal with a macrolide ${ }^{11}$ Roxithromycin has been shown to reduce oedema formation with an effect almost equal to that of the non-steroidal anti-inflammatory drug nimesulide, while azithromycin and clarithromycin showed lesser anti-inflammatory effects. Roxithromycin has been further evaluated in a variety of rat models including poly-L-arginine induced paw oedema, croton oil inflamed ear assay, and intraperitoneal polyester sponge granuloma ${ }^{12}$ It produced a marked anti-oedema effect similar to that of indomethacin in poly-L-arginine assay, significant inhibition of croton oil induced inflammation in the ear, but failed to reduce the development of granuloma induced by implanted polyester sponges. Pretreatment with erythromycin decreased neutrophil counts in BAL fluid from the lungs of mice in which inflammation had been

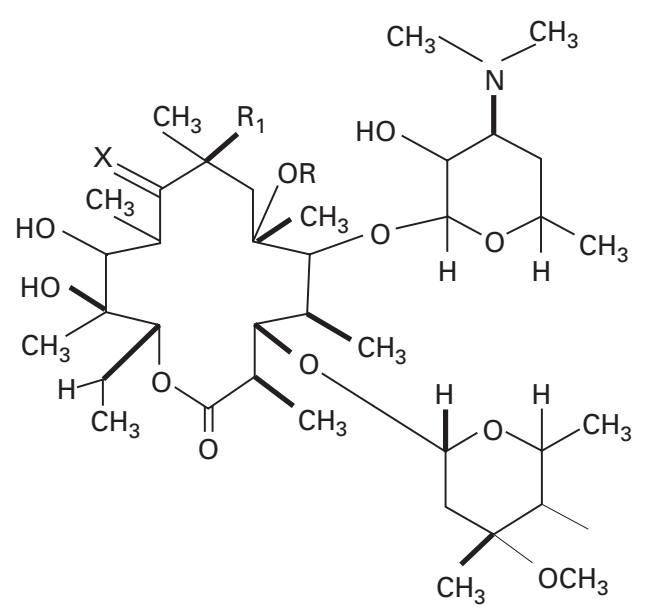

\begin{tabular}{|l|lll|}
\hline & $\mathrm{X}$ & $\mathrm{R}$ & $\mathrm{R}_{1}$ \\
\hline Erythromycin A & $\mathrm{O}$ & $\mathrm{H}$ & $\mathrm{H}$ \\
Clarithromycin & $\mathrm{O}$ & $\mathrm{CH}_{3}$ & $\mathrm{O}$ \\
Roxithromycin & $\mathrm{NOCH}_{2} \mathrm{O}\left(\mathrm{CH}_{2}\right)_{2} \mathrm{OCH}_{3}$ & $\mathrm{H}$ & $\mathrm{H}$ \\
\hline
\end{tabular}

Figure 1 Fourteen-membered macrolide ring structure.

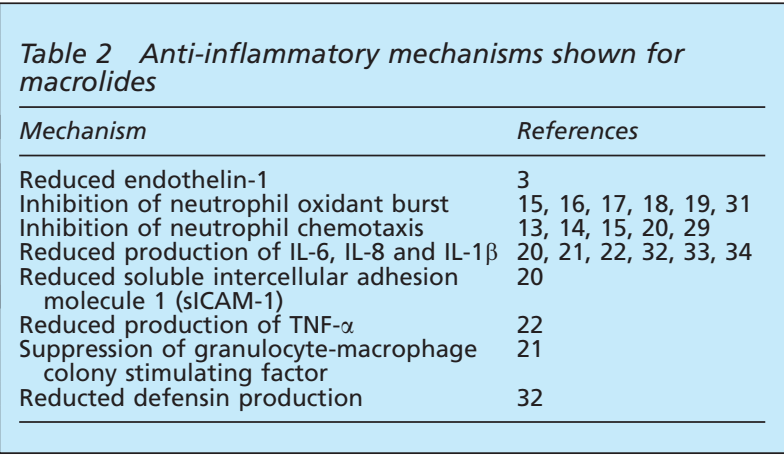

induced by intratracheal instillation of lipopolysaccharid $\left.\right|^{13}$ or gerosolised Proteus mirabilis and Staphylococcus aureus..$^{14}$ Thus, an anti-inflammatory effect has been repeatedly demonstrated in animal models with of roxithromycin, seemingly more potent than azithromycin and clarithromycin, with erythromycin having 8 the least effect. It has been postulated that this could be due to better cell penetration by the newer macrolides than by erythromycin.

\section{Macrolides and the neutrophil oxidant burst}

Reactive oxidant products of neutrophils are known to $\frac{c}{\omega}$ damage tissue and the intracellular accumulation of $\overrightarrow{\vec{\varphi}}$ macrolides may limit their production, although data on this are conflicting. Lambro et $\mathrm{d}^{1 \text { 15 }}$ have shown that only roxithromycin strongly decreased the polymorphonuclear neutrophil (PMN) oxidative burst as assessed by luminol amplified chemiluminescence, $\bar{\partial}$ superoxide anion generation, and myeloperoxidase mediated iodination of proteins. This effect was noted to $\stackrel{\mathbb{Z}}{2}$ vary significantly between individuals and may relate to $\overrightarrow{\overrightarrow{0}}$ the high concentration of roxithromycin achieved within 3 the neutrophil. Anderson et $\mathrm{dl}^{\mathrm{l}}$ found that both erythromycin and roxithromycin selectively inhibited superoxide generation by activated neutrophils. Hand et $\mathrm{a} l^{17}$ observed that roxithromycin which readily enters the phagocyte was an efficient inhibitor of the $\stackrel{x}{\sigma}$ PMN superoxide generation stimulated by formyl- 3 . methionyl-leucyl-phenylalanine (FMLP) and concanavalin A. Clearly, it would be of interest to minimise the oxidative response of human PMNs whilst preserving their bactericidal and phagocytic functions.

\section{Macrolides and neutrophil chemotaxis}

Stimulation of neytrophil migration has been described $\mathbb{N}$ in two studies. ${ }^{1 / 18}$ In one, adult volunteers were given $\underset{\omega}{N}$ a single oral dose of $500 \mathrm{mg}$ erythromycin stearate and a significant increase in PMNL migration in response 6 to a leucocyte attractant was observed at 90 minutes. Conflicting data were produced in a study by Torre et $\stackrel{+}{+}$ af ${ }^{19}$ who observed decreased PMN chemotaxis following $\frac{7}{0}$ the ingestion of erythromycin, josamycin, miokamycin, $\overrightarrow{\mathbb{D}}$ roxithromycin, and rokitamycin for four days by adult $\frac{\rho}{\mathbb{Q}}$ volunteers. Reduction in chemotaxis was observed by $\Omega$ other workers $\left.\right|^{1} \mid{ }^{14}$ raising concerns that the bactericidal effects of these antibiotics might be reduced. However, 응 the relevance of these findings in the clinical setting is not yet known.

\section{Macrolides and cytokine production}

Cytokines are small proteins involved in the orchestration of the inflammatory process. They can be either pro-inflammatory (for example, tumour necrosis 
factor (TNF) alpha, interleukin (IL)-6, IL-8 and IL12, and gamma interferon) or anti-inflammatory (for example, IL-10). Macrolides, particularly those derived from erythromycin $\mathrm{A}$, have been shown to impair the production of pro-inflammatory cytokines. Haemophilus influenzae induces the release of IL-6, IL-8, and soluble intercellular adhesion molecule 1 (sICAM-1) from airway epithelial cells and this effect can be reduced by erythromycin. ${ }^{20}$ Similarly, roxithromycin has been shown to suppress the production of IL- 6 and IL- 8 and granulocyte-macrophage colony stimulating factor, in addition to inhibiting neutrophil adhesion to epithelial cells. ${ }^{21}$ Erythromycin also caused a dose dependent decrease in heat killed Streptococcus pneumoniae (HKSP) induced production of TNF- $\alpha$ and IL- 6 in human whole blood in vitro. ${ }^{22}$ The production of IL-1, IL-12, and gamma interferon was only affected at the highest concentration of erythromycin. The production of TNF- $\alpha$ and IL-6 in whole blood obtained from healthy subjects after a 30 minute infusion of $1 \mathrm{~g}$ of erythromycin was lower after ex vivo stimulation with HKSP than blood drawn before infusion. Effects on the cytokine pathways are complex but reduction in the pro-inflammatory cytokines in experimental models provides some insight into how these effects might be achieved in vivo.

\section{Macrolides and asthma}

Macrolides have been shown to affect bronchial hyperresponsizeness by a non-antibiotic mechanism. Rosenberg et a ${ }^{23}$ described a patient with corticosteroid dependent asthma in whom the addition of daily troleandomycin allowed corticosteroids to be weaned without clinical deterioration (table 3 ). Erythromycin has been shown to reduce the severity of bronchial hyperresponsiveness in adult asthmatics who were not corticosteroid dependen ${ }^{24}, 200 \mathrm{mg}$ of erythromycin three times a day given over a 10 week period resulted in a significant increase in the $\mathrm{PC}_{20}$ in both atopic and non-atopic patients, supporting the observation of an improvement in asthma control by macrolides by workers in the early 1970s. The interaction of erythromycin with theophylline, reducing its clearance and increasing plasma theophylline levels, could partly explain the beneficial effects of erythromycin. However, in this study $600 \mathrm{mg}$ erythromycin daily produced no change in serum theophylline levels. A further study was performed on children with asthma using roxithromycin, an antibiotic with little effect on the pharmacokinetics of theophylline. ${ }^{2}$ Again, the $\mathrm{PC}_{20}$ significantly increased with $150 \mathrm{mg}$ roxithromycin daily after four and eight weeks of treatment. This study also reported no change in the liver enzymes SGOT and SGPT or morning cortisol levels and concluded that roxithromycin did not affect corticosteroid metabolism.

Reduced production of reactive oxygen species by polymorphomuclear leucocytes or an effect on neutrophil chemotaxis or cytokine production may be involved. Decreased production of neutrophil chemotactic lymphokines has also been postulated ${ }^{14}$ Experimentally, erythromycin has been shown to reduce the electrical field stimulation induced contraction of isolated human bronchial strips in a dose dependent fashion, suggesting that macrolides may inhibit the cholinergic neuroeffector mechanism possibly by reducing acetylcholine release at nerve terminals ${ }^{26}$ However, the effect on endothelin-1 described in the Introductory Article is likely to be another important mechanism by which bronchoconstriction is reduced.

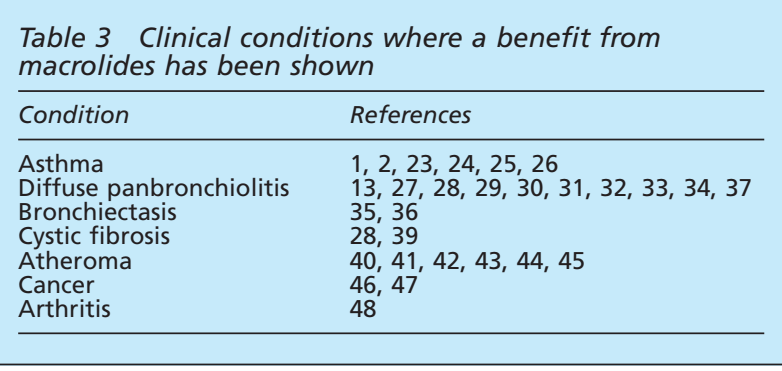

Macrolides and diffuse panbronchiolitis (DPB)

A clinical role for macrolides, which is not due to a direct antimicrobial effect, is perhaps best shown in $\vec{\circ}$ DPB. This disease is characterised by chronic bronchial $\overrightarrow{\vec{H}}$ sepsis and airflow obstruction with chronic inflammation of the respiratory bronchioles, stenoses, and $\vec{x}$ obstruction. Initial infections are with H influenzae, Strep or pneumoniae, and Staph aureus, and eventually patients become colonised with Pseudomonas aeruginosa. Death 8 due to respiratory failure occurs after repeated cycles of $\infty$ infection. The disease occurs commonly in Japan with on occasional cases reported in Italy and North America. The prognosis of DPB has been dramatically improved in recent years by the use of erythromycin and other $\vec{\nabla}$ macrolide antibiotics in this condition. In 1984 the five year survival rate was only $26 \%$ in cases with Paeruginosa क् and $55 \%$ for all other types of DPB. Since erythromycin $\overrightarrow{\vec{\theta}}$ has become widely used, the 10 year survival for all $\&$ types of DPB has increased to $94 \%$.

The clinical efficacy of erythromycin in this condition was first noticedin 1982 and has since been confirmed by clinical trial $\$^{22}$ but the mechanism of action is still unknown. Studies indicate a crucial role for the $\frac{\mathrm{Q}}{\mathrm{D}}$ polymorphonuclear leucocyte in the pathogenesis with $\varrho$ influx of these cells into the airways and the production of oxidants and proteolytic enzymes producing in- 3 flammatory change. Bronchoalveolar lavage (BAL) fluid from sufferers has demonstrated increased numbers of neutrophils, neutrophil derived elastolytic-like activity, $\overrightarrow{\vec{\partial}}$ IL-8, IL-1 $\beta$, and leukotriene B4 (LTB4). After treatment with erythromycin the number of neutrophils and amount of neutrophil derived elastolytic-like activity in 3 BAL fluid has been shown to decrease significantly with 8 a coincident improvement in lung function ${ }^{29}$ This was also described by Kadota et a 13 who found increased 을 numbers of neutrophils and neutrophil chemotactic activity in the BAL fluid of patients compared with normal subjects which was reduced by erythromycin N treatment; this was also found in an animal model. Benefit is not confined to erythromycin and has been 0 demonstrated with other 14-membered macrolides. An $\mathbf{\omega}$ efficacy of $79 \%$ for erythromycin $(400 \mathrm{mg}$ or $600 \mathrm{mg}$ ), 우 $86 \%$ for roxithromycin ( $150 \mathrm{mg}$ or $300 \mathrm{mg}$ ), and $67 \%$ 을 for clarithromycin (200 mg or $400 \mathrm{mg}$ ) taken daily for $\mathbb{\Phi}$ at least two months has been reported ${ }^{30}$ Azithromycin, $\stackrel{+}{+}$ a 15-membered macrolide, has also been shown to $\frac{0}{0}$ be effective ${ }^{31}$ Defensins, antimicrobial and cytotoxic peptides which occur in high concentrations in the BAL $\stackrel{?}{\mathbb{D}}$ fluid of patients with DPB are reduced with macrolide 응 treatmen ${ }^{32}$ and several studies haye shown a reduction

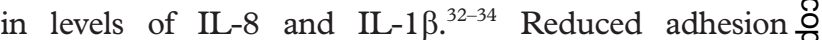
molecule macrophage activating complex 1 (MAC-1) on peripheral blood neutrophils has also been shown to occur $\frac{\bar{Q}}{\bar{D}}$ after macrolide therapy ${ }^{33}$ Sixteen-membered macrolides? including josamycin are not beneficial in DPB.

Macrolides and bronchiectasis/cystic fibrosis

The dramatic effect of macrolides on the prognosis of 
DPB has led to trials in other conditions characterised by chronic bronchial sepsis including bronchiectasis and cystic fibrosis. Both conditions are associated with copious sputum production, rhinosinusitis, progressive airway destruction, and chronic $P$ aerurginosa infection of the airways. A double blind, placebo controlled trial of low dose erythromycin in bronchiectasis has recently been published..$^{3}$ Erythromycin was given to 11 patients with bronchiectasis in a dose of $500 \mathrm{mg}$ twice daily for an eight week period and $\mathrm{FEV}_{1}$, forced vital capacity (FVC), and sputum volume over 24 hours were significantly improved compared with placebo. However, no parallel improvement in sputum pathogens, leucocytes, IL- $1 \alpha$ and IL-8, TNF- $\alpha$ or LTB 4 was found. Erythromycin is unlikely to be bactericidal in view of the low dosage and poor penetration into the bronchial tree, and the authors postulate that inhibition of glycoconjugate release and chloride secretion by airway epithelium and macrophage mucus secretagogue production might result in decreased sputum water content and volume. Airway responsiveness in bronchiectasis has also been shown to improve with long term, low dose macrolide administration. Roxithromycin was given to 13 children with increased airway responsiveness and bropchiectasis and a group of 12 controls received placebo ${ }^{36}$ Methacholine challenge tests were performed at baseline and after 12 weeks. The $\mathrm{PC}_{20}$ increased significantly in the roxithromycin group while no change was seen in the placebo group. In addition, improvement in sputum features was noted after six weeks in the treatment group.

That macrolides might be efficacious in these conditions by a non-bactericidal effect on $P$ aeruginosa has been postulated by Howe and Spencer ${ }^{37}$ Diffuse panbronchiolitis, bronchiectasis, and cystic fibrosis are all associated with chronic $P$ aeruginosa infection and inhibition of this organism could be a mode of action. Two mechanisms by which macrolides might be effective are proposed: an effect on the immune system to modify the inflammatory response to infection or a direct effect on $P$ aeruginosa to decrease its virulence. In addition to the effects on the immune system described above, macrolides inhibit endotoxin A, total protease, elastase, phospholipase C, DNase, lecithinase, gelatinase, lipase, pyocyanin and motility, all virulence factors associated with $P$ aeruginosa infection. Strains of $P$ aeruginos a found in these conditions produce a mucoid alginate by which they closely adhere to the airway surface. In this "biofilm" the bacteria are resistant to attack by antibacterial agents and interaction with neutrophils. The alginate works as an antigen and serum titres of anti-alginate antibody IgG and IgA are significantly higher in Pseudomonas positive patients with DPB than in Pseudomonas negative patients. In experimental models the alginate induced antigen-antibody reaction resulted in lympho- cyte infiltration around small airways which gradually developed into granuloma-like infiltration containing macrophages. The state of antigen excess resulting from persistent colonisation of mucoid alginate producing $P$ F aeruginosa may generate an immune complex in the 윽 host. The levels of serum immune complexes in patients $\underset{x}{x}$ with DPB have been positively correlated with clinical $\vec{\Rightarrow}$ symptoms. These immune complexes deposit on lung $\stackrel{\text { क }}{9}$ tissue and stimulate neutrophil chemotaxis ${ }^{38}$ Long term treatment with clarithromycin has resulted in most cases $\frac{\bar{O}}{\bar{D}}$ in a decrease in serum immune complexes and an improvement in clinical symptoms. ${ }^{2}$ Erythromycin, $\cong$ clarithromycin, and azithromycin have been shown to क be effective inhibitors of antigen-antibody reaction in $\overrightarrow{0}$ vitro but not 16-membered macrolides or ce- $\overrightarrow{\vec{H}}$ phalosporins. Long term treatment with azithromycin $\vec{\omega}$ has been shown to improve lung function in patients with cystic fibrosis 39

Combining macrolides with antipseudomonal anti- or biotics may be more effective than administering anti- $N$ pseudomonal antibiotics alone 28 In vitro incubation of $\varnothing_{\infty}$ biofilm $P$ aeruginosa with twice the minimum bactericidal of concentration of ciprofloxacin resulted in $85 \%$ of bacteria $\infty$ remaining viable. However, when clarithromycin or 을 azithromycin was added, the bactericidal effect of cipro- floxacin was far greater. This offers new prospects for clinical trials of these antibiotics in combination in patients with cystic fibrosis and bronchiectasis.

Atheroma, arthritis, and cancer

An effect of macrolides has been described in each of these conditions. Whether the mechanism relates to its anti-inflammatory effect, antimicrobial effect, or some ڤँ other effect remains to be discovered. The development $\stackrel{\circ}{\mathbb{D}}$ of atheroma has been linked to chronic chlamydial $\underset{\vec{z}}{\vec{a}}$ infection $4{ }^{43}$ Two clinical trials have been undertaken $\frac{0}{3}$ of macrolide therapy as secondary prevention in patients with known coronary artery disease. In the first by Gupta et af ${ }^{44} 220$ male survivors of acute myocardial infarction were screened for IgG antibody to Chlamydia 음 pneumoniae by microimmunoflorescence. Those with titres of $>1 / 64$ on two consecutive occasions were ran- $\dot{\sigma}$ domised to receive azithromycin $500 \mathrm{mg}$ daily for three days and repeated at three months or placebo. Patients $\delta$ were followed for a mean of 18 months. A five fold $₹$ reduction in adverse cardiovascular events was found $\mathrm{O}$ $(\mathrm{p}=0.03)$ in the azithromycin treated group compared $>$ with placebo. A second study by Gurfinkel et a $l^{4}$ enrolled 202 patients with unstable angina or non-Q wave myo- $N$ cardial infarction. Patients were randomised to receive $\sigma$ roxithromycin $150 \mathrm{mg}$ twice daily for 30 days or placebo, 옹 independent of $C$ pneumoniae serostatus. They were $\mathbf{\omega}$ followed for six months and the primary end point was a composite of three outcomes - cardiac death, acute $\stackrel{0}{\varnothing}$

\section{LEARNING POINTS} * Multiple in vitro and in vivo anti-inflammatory effects of macrolides have been dem- $\frac{\stackrel{\Omega}{\mathbb{D}}}{2}$
onstrated.

* Anti-inflammatory effects appear to be limited to macrolides with 14-membered and 15membered ring structure.

* A clear clinical role for macrolides has so far only been shown in DPB.

* Future work should focus on the molecular mechanisms of the macrolide-cell interaction leading to the development of new, more specific, anti-inflammatory macrolides.

* Inhibition of endothelin-1 may be partly responsible for an anti-asthma effect. 
myocardial infarction, and severe recurrent ischaemia; $2 \%$ of the roxithromycin group and $9 \%$ of the placebo group reached the triple end point $(p=0.032)$. The double end point of myocardial infarction or cardiac death was reached by $4 \%$ in the placebo group and by none of the roxithromycin treated group $(p=0.058)$. An association between $C$ pneumoniae and coronary artery disease has been shown but whether the relationship is causal or not is unknown. These two small studies of macrolides in secondary prevention will stimulate further studies in this field.

Helicobacter pylori, another macrolide susceptible organism, may also play a role in coronary artery disease but is definitely responsible for gastric and duodenal ulcers and plays an important part in the development of gastric cancer. Eradication therapy is an appropriate way of treating gastric and duodenal ulcers and clarithromycin in combination with another antibiotic and a proton pump inhibitor is usually employed. Whether there will be a reduction in the incidence of gastric cancer remains to be shown.

An anti-cancer effect of clarithromycin has been demonstrated in various aminal model $\beta^{4} \mid 7$ but efficacy in man has yet to be demonstrated.

A possible role for macrolides in arthritis has been considered but further research is needed ${ }^{48}$

\section{Conclusions}

Multiple in vitro and in vivo anti-inflammatory effects of macrolides have been demonstrated. As the complex molecular interactions which determine the inflammatory cascade become better understood, it is likely that more will be found. A clear clinical role for macrolides has so far only been shown in DPB. Future work should focus on the molecular mechanisms of macrolide-cell interaction leading to the development of new, more specific, anti-inflammatory macrolides. The door is open for further clinical trials of macrolides in a number of clinical areas.

1 Itkin IH, Menzel ML. The use of macrolide antibiotic substances in the treatment of asthma. F Allergy 1970;45:146-62.

2 Miatake H, Taki F, Taniguchi H, et al. Erythromycin reduces the severity of bronchial hyperresponsiveness in asthma. Chest 1991;99:670-3.

3 Takizawa H, Desaki M, Ohtoshi T, et al. Erythromycin and clarithromycin attenuate cytokine-induced endothelin-1 expression in rithromycin attenuate cytokine-induced endothelin-1 expres
human bronchial epithelial cells. Eur Respir 7 1998;12:57-63.

4 Mangisawa $M$, Kurihara H, Kimura S, et al. A novel potent vasoconstrictor peptide produced by vascular endothelial cells. Nature
chisa 1988;332:411-5.

5 Advenier C, Sarria B, Naline E, et al. Contractile activity of three endothelins (ET-1, ET-2 and ET-3) on the human isolated bronchus. Br f Pharmacol 1990;100:168-72.

6 Mura S, Ishiara SH, Satoh M, et al. Endothelin regulation of mucus glycoprotein secretion from feline tracheal submucosal glands. $A m \mathcal{F}$ Physiol 1992;262:L208-13.

7 Sirois MG, Filep JG, Rouseau A, et al. Endothelin-1 enhances vascular permeability in conscious rats: role of thromboxane A2. Eur $\mathcal{F}$ Pharmacol 1992;214:119-25.

8 Finsnes F, Skjonsberg OH, Tonnessen T, et al. Endothelin production and effects of endothelin antagonism during experimental airway inflammation. Am Rev Respir Crit Care Med 1997;155:1404-12.

9 Mattoli S, Mezzetti M, Riva G, et al. Specific binding of endothelin on human bronchial smooth muscle cells in culture and secretion of endothelin-like material from bronchial epithelial cells. Am f Respir endothelin-like material from

10 Vittori I, Marini M, Fasoli A, et al. Increased expression of endothelin in bronchial epithelial cells of asthmatic patients and effect of corticosteroids. Am Rev Respir Dis 1992;146:1320-5.

11 Scaglione F, Rossoni G. Comparative anti-inflammatory effects of roxithromycin, azithromycin and clarithromycin. $\mathcal{F}$ Antimicrob Chemother 1998;41(Suppl B): 47-50

12 Agen C, Danesi R, Blandizzi C, et al. Macrolide antibiotics as antiinflammatory agents: roxithromycin in an unexpected role. Agents Actions 1993;38:85-90.

13 Kadota J, Sakito O, Kohno S, et al. A mechanism of erythromycin treatment in patients with diffuse panbronchiolitis Am Rev Respir Dis 1993;147:153-9.

14 Nelson S, Summer WR, Terry PB, et al. Erythromycin-induced suppression of pulmonary antibacterial defenses. A potential mechanism

15 Lambro MT, El Benna J, Babin-Chevaye C. Comparison of the invitro effect of several macrolides on the oxidative burst of human neutrophile. F Antimicrob Chemother 1989;24:561-72.
16 Anderson R. Erythromycin and roxithromycin potentiate human neutrophil locomotion in vitro by inhibition of leukoattractant activated phil locomotion in vitro by inhibition of leukoattractant activated

17 Hand WL, Hand D, King-Thompson N. Antibiotic inhibition of the respiratory burst response in human polymophonuclear leukocytes. Antimicrob Agents Chemother 1990;34:863-70.

18 Anderson R, Fernandes AC, Eftychis HE. Studies on the effects of $\overline{0}$ ingestion of a single $500 \mathrm{mg}$ oral dose of erythromycin stearate on leucocyte motility and transformation and on release in vitro of $\times$ prostaglandin E2 by stimulated leucocytes. I Antimicrob Chemother $\Rightarrow$ 1984;14:41-50.

19 Torre $\mathrm{D}$, Broggini $\mathrm{M}$, Botta $\mathrm{V}$, et al. In vitro and ex vivo effects of recent and new macrolide antibiotics on chemotaxis of human polymorphonuclear leukocytes. Chemotherapy 1992;3:236-9.

20 Khair OA, Devalia JL, Abdelaziz MM, et al. Effect of erythromycin on

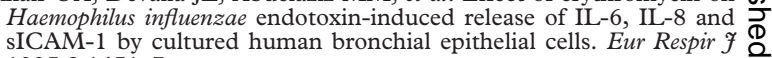
1995;8:1451-7.

21 Kawasaki S, Takizawa H, Takayuki O, et al. Roxithromycin inhibits cy- ڤొ tokine production by and neutrophil attachment to human bronchial epithelial cells in vitro. Antimictob Agents Chemother 1998;42:1499-502.

22 Schultz, M, Speelman P, Zaat S, et al. Erythromycin inhbits tumour necrosis factor alpha killed and interleukin 6 production induced by $\vec{\omega}$ heat-killed Streptococcus pneumoniae in whole blood. Antimicrob Agents Chemother 1998;42:1605-9.

23 Rosenberg SM, Gerhand H, Grunstein MM, et al. Use of TAO without methylprednisolone in the treatment of severe asthma. Chest 1991; of
100:849-50.

24 Miyatake $\mathrm{H}$, Taki $\mathrm{F}$, Taniguichi $\mathrm{MD}$, et al. Ethrythomycin reduces the $\mathrm{N}$ severity

25 Shimizu T, Masahiko K, Mochizuki H, et al. Roxithromycin reduces the degree of bronchial hyperresponsiveness in children with asthma. Chest 1994;106:458-61.

26 Tamaoki J, Tagaya E, Sakai A, et al. Effects of macrolide antibiotics on neurally mediated contraction of human isolated bronchus. $\mathcal{F}$ Allergy Clin Immunol 1995;95:853-9.

27 Yamamoto $M$, Kondo A, Ramura $M$, et al. Long-term therapeutic effects of erythromycin and new quinolone antibacterial agents on diffuse panbronchiolitis. Fapanese f Thorac Dis 1990;28:1305-13.

28 Kobayashi H. Biofilm disease: its clinical manifestation and therapeutic $\bar{\oplus}$ possibilities of macrolides. Am f Med 1995;99(Suppl 6A):26-30S.

29 Ichikawa Y, Ninomiya H, Koga H, et al. Erythromycin reduces neutro- $\vec{\bullet}$ phils and neutrophil-derived elastolytic-like activity in the lower respiratory tract of bronchiolitis patients. Am Rev Respir Dis 1992;146: 196-203.

30 Shirai T, Sato A, Chida K. Effect of 14-membered ring macrolide therapy on chronic respiratory tract infection and polymorphonuclear activity. Intern Med 1995;34:469-74.

31 Kobayashi H, Shimada K, Nakata K, et al. Study on azithromycin in treatment of diffuse panbronchiolitis. In: Programme and Abstracts of the Third International Conference on the Macrolides, Azalides and Streptogranins, Lisbon, 1996: Abstract 912, p 75.

32 Ashitani J, Mukae H, Nakazato $M$, et al. Elevated concentration of $\overrightarrow{\bar{O}}$ defensins in bronchoalveolar lavage fluid in diffuse panbronchiolitis. Eur Respir F 1998;11:104-11.

33 Mukae H, Kadota J, Ashitani J, et al. Elevated levels of soluble adhesion molecules in serum of patients with diffuse panbronchiolitis. Chest 1997;112:1615-21

34 Takizawa H, Deskai M, Ohtoshi T, et al. Erythromycin modulates IL8 expression in normal and inflamed human bronchial epithelial cells. Am Rev Respir Dis 1997;156:266-71.

35 Tsang KWT, Ho PI, Chan Kn, et al. A pilot study of low-dose erythromycin in bronchiectasis. Eur Respir f 1999;13:361-4.

36 Koh YY, Lee MH, Sun YH, et al. Effect of roxithromycin on airway responsiveness in children with bronchiectasis: a double-blind, responsiveness in children with bronchiectasis: a

37 Howe RA, Spencer RC. Macrolides for the treatment of Pseudomonas aeruginosa infections? If Antimicrob Chemother 1997;40:153-5.

38 Hoiby N, Doring G, Sehoitz PD. The role of immune complexes in the pathogenesis of bacterial infections. Ann Rev Microbiol 1986;40:29-53. 39 Jaffe A, Francis J, Rosenthal M, et al. Long-term azithromycin may improve lung function in children with cystic fibrosis. Lancet 1998; 351:420

40 Patel P, Mendall MA, Carrington D, et al. Association of Helicobacter 5 pylori and Chlamydia pneumoniae with coronary heart disease and $\mathbf{N}$ cardiovascular risk factors. BMF 1995;311:711-4.

41 Blasi F, Cosentini R, Raccanelli R, et al. A possible association of $\mathrm{\omega}$ Chlamydia pneumoniae infection and acute myocardial infarction in $\mathbf{\sigma}$ patients younger than 65 years of age. Chest 1997;112:309-12.

42 Kuo CC, Shor A, Cambell LA, et al. Demonstration of Chlamydia 0 pneumoniae in atherosclerotic lesions of coronary arteries. F Infect Dis pneumoniae in at

43 Cambell LA, O'Brien ER, Cappuccio AL, et al. Detection of Chlamydia pneumoniae TWAR in human coronary atherectomy tissues. 7 Infect pneumoniae TWAR in human coronary atherectomy tissues. F Infect $\frac{0}{0}$ Dis 1995;172:585-8.

44 Gupta S, Leatham EW, Carrington D, et al. Elevated levels of Chlamydia pneumoniae antibodies, cardiovascular events and azithromycin in male survivors of myocardial infarction. Circulation 1997;96:404-7

45 Gurfinkel E, Bozovich G, Daroca A, et al (ROXIS Study Group). Randomized trial of roxithromycin in non-Q-wave coronary syndromes: ROXIS pilot study. Lancet 1997;350:404-7.

46 Sassa K, Mizushima Y, Fujishita T, et al. Clarithromycin: effect of a new macrolide antibiotic on a transplanted tumour in rats. Programme and Abstracts of the 4th International Conference on the Macrolides, Azalides, Streptogramins and Ketolides, 21-23 fanuary 1998, Barcelona, Spain. Abstract 4-03.

47 Shibuya M, Kobubo Y, Hino M, et al. Effect of clarithromycin on experimental lung metastasis. Programme and Abstracts of the 4th International Conference on the Macrolides, Azalides, Streptogramins and Ketolides, 21-23 Fanuary 1998, Barcelona, Spain. Abstract 4-03.

48 Arayssi T Is there a role for macrolides in arthritis. Programme and Abstracts of the 4th International Conference on the Macrolides, Azalides, Streptogramins and Ketolides, 21-23 fanuary 1998, Barcelona, Spain. Abstract 6. 